\title{
GENERALIZED RANK ANNIHILATION METHOD. II: BIAS AND VARIANCE IN THE ESTIMATED EIGENVALUES
}

\author{
N. M. FABER, L. M. C. BUYDENS AND G. KATEMAN \\ Deparmem of Analyical Chemistry, University of Nijmegen, Toernooiveld 1, NL-6525 ED Nijmegen,
} Netheriands

\begin{abstract}
SUMMARY
Rank annihilation factor analysis (RAFA) is a method for multicomponent calibration using two data matrices simultaneously, one for the unknown and one for the calibration sample. In its most general form, the generalized rank annihilation method (GRAM), an eigenvalue problem has to be solved. In this second paper expressions are derived for predicting the bias and variance in the eigenvalues of GRAM. These expressions are built on the analogies between a reformulation of the eigenvalue problem and the prediction equations of univariate and multivariate calibration. The error analysis will also be performed for Lorber's formulation of RAFA. It will be demonstrated that, depending on the size of the eigenvalue, large differences in performance must be expected. A bias correction technique is proposed that effectively eliminates the bias if the error in the bias estimate is not too large. The derived expressions are evaluated by Monte Carlo simulations. It is shown that the predictions are satisfactory up to the limit of detection. The results are not sensitive to an incorrect choice of the dimension of the factor space.
\end{abstract}

KEY WORDS RAFA GRAM Eigenvalues Bias Variance

\section{INTRODUCTION}

RAFA is a calibration and curve resolution technique that enables the quantification of a component in the presence of an unknown background. ${ }^{1}$ The method can be used if the signal of the analytes of interest is bilinear and identical in the unknown and calibration sample. Furthermore, the analytes of interest should raise the rank of the data matrices by one. The model is represented as

$$
\begin{aligned}
& \mathbf{M}=\mathbf{X C}_{\mathrm{M}} \mathbf{Y}^{\mathrm{T}}=\mathbf{H} \mathbf{Y}^{\mathrm{I}} \\
& \mathbf{N}=\mathbf{X C _ { N }} \mathbf{Y}^{\mathbf{T}}=\mathbf{H} \Pi \mathbf{Y}^{\mathbf{T}}
\end{aligned}
$$

where $\mathbf{M}$ and $\mathbf{N}$ denote the unknown and calibration data matrices respectively. The pure component profiles $\mathbf{X}$ and $\mathbf{Y}$ are normalized and the diagonal matrices $\mathbf{C}_{\mathbf{M}}$ and $\mathbf{C}_{\mathbf{N}}$ contain the scaling factors. In the following we will assume that the calibration sample does not contain components that are missing in the unknown sample. In that case it is possible to absorb the matrix $\mathbf{C}_{M}$ into the scaled profiles. The conventional choice is to denormalize the column profiles leading to the matrices $\mathbf{H}=\mathbf{X} \mathbf{C}_{\mathbf{M}}$ and $\boldsymbol{\Pi}=\mathbf{C}_{\mathbf{M}}^{-1} \mathbf{C}_{N}$. In order to resolve (the relevant part of) the data matrices and obtain the corresponding concentration ratios $\Pi$, the data matrices are combined in one equation. This can be accomplished in several ways. Representing $\mathbf{M}$ by

CCC 0886-9383/94/030181-23

Received 19 July 1993

(c) 1994 by John Wiley \& Sons, Ltd. Accepted 12 October 1993 
the significant PCs

$$
\overline{\mathbf{M}}=\overline{\mathbf{A}} \overline{\mathbf{B}}^{\mathrm{T}}=\overline{\mathbf{U}} \overline{\boldsymbol{\theta}} \overline{\mathbf{V}}^{\Upsilon}
$$

leads to the following decompositions of $\Pi:^{2}$

$$
\begin{aligned}
\Pi & =\mathbf{H}^{+} \mathbf{N}\left(\mathbf{Y}^{\mathrm{T}}\right)^{+} \\
& =\mathbf{T}^{-1}\left(\overline{\mathbf{A}}^{+} \mathbf{N} \overline{\mathbf{B}}\right) \mathbf{T} \\
& =\mathbf{T}^{-1}\left(\overline{\boldsymbol{\Theta}}^{-1} \overline{\mathbf{U}}^{\mathrm{T}} \mathbf{N} \overline{\mathbf{V}}\right) \mathbf{T} \\
& =\mathbf{Z}^{-1}\left(\overline{\mathbf{U}}^{\mathrm{T}} \mathbf{N} \overline{\mathbf{V}} \overline{\boldsymbol{\theta}}^{-1}\right) \mathbf{Z}
\end{aligned}
$$

where $\mathbf{A}, \mathbf{B}, \mathbf{U}$ and $\mathbf{V}$ denote the scores, loadings and left and right singular vectors respectively, $\boldsymbol{\theta}$ is the diagonal matrix with singular values and the matrices $\mathbf{T}$ and $\mathbf{Z}$ contain eigenvectors. The 'overbar' indicates that the decomposition in equation (2) is truncated. Equations (3b)-(3d) are eigenvalue problems that directly provide the desired information, whereas equation (3a) shows the relation to the physical decomposition of the data matrices. Equation ( $3 \mathrm{~d}$ ) was first derived by Lorber ${ }^{3}$ for the case where the calibration sample contains only one component. The generalization of his method is achieved by constructing a factor space that spans both data matrices. Sánchez and Kowalski ${ }^{4}$ have solved this problem by decomposing the sum matrix and recently Wilson et al. ${ }^{5}$ have recommended decomposing the row and column adjoined matrices.

The decomposition in real profiles has a clear interpretational advantage over the decomposition in abstract profiles. The presence of the pseudoinverse matrices makes the relation with multivariate calibration obvious. From multivariate calibration it is well known that the pseudoinverse of a stochastic calibration matrix will lead to biased concentration estimates. ${ }^{6}$ It follows that the eigenvalues must be expected to be biased also. In order to derive expressions for the prediction of bias it will therefore be advantageous to apply the results already obtained for multivariate calibration. Furthermore, we have recently derived standard errors in the eigenvalues by performing first-order error propagation on equation ( $3 \mathrm{~d}){ }^{7}$ In the derivation we make use of approximations that are more transparent when error propagation is performed on equation (3a). We will derive expressions for bias and variance in the eigenvalues for Lorber's method, the generalization of Sánchez and Kowalski and the generalization of Wilson et al. If possible, the expressions will be presented in the same way as already known for multivariate calibration. Bias and variance resulting from errors in the concentrations can be predicted from results derived for univariate calibration. ${ }^{8}$

The remaining part of this paper will be organized as follows. First we will give a general introduction to the effect of random errors on an estimated parameter. Next we will derive the bias expressions for the eigenvalues. In the following section we will summarize the relevant expressions for the variance in the eigenvalues. Next we will introduce a procedure for the correction of bias. This procedure should perform well if the bias estimate is reasonable. Next we will show how these bias-corrected eigenvalues can be used to set up a probability range. In the following sections we will briefly discuss the bias and variance in the unknown concentrations. It will be shown from the variance expression for the unknown concentration that for the methods discussed the generalization of Sanchez and Kowalski will always give the smallest (relative) variance in the eigenvalues. Finally, the adequacy of the derived expressions is tested by performing Monte Carlo simulations. We will restrict ourselves to examining the effect of random noise in the response matrices, since the effect of noise in the concentrations is trivial. It will be shown that the expressions are reliable up to the limit of detection. Furthermore, the results do not seem to be sensitive to the choice of dimensionality of the factor space. 


\section{NOTATION AND CONVENTIONS}

Boldface uppercase letters represent matrices, e.g. A. For a given matrix $\mathbf{A}$ the matrices $\mathbf{A}^{\mathrm{T}}$, $\mathbf{A}^{-1}$ and $\mathbf{A}^{+}$stand for its transpose, inverse and pseudoinverse respectively. The 'inverse transpose' and 'pseudoinverse transpose' matrices will be denoted by the shorthand notation $\mathbf{A}^{-\mathrm{T}}=\left(\mathbf{A}^{-1}\right)^{\mathrm{T}}=\left(\mathbf{A}^{\mathrm{T}}\right)^{-1}$ and $\mathbf{A}^{+}=\left(\mathbf{A}^{+}\right)^{\mathrm{T}}=\left(\mathbf{A}^{\mathrm{T}}\right)^{+}$. The matrix element in row $i$ and column $j$ of $\mathbf{A}$ will be specified by a row and column index as $A_{i j}$. The $n$th row and $n$th column of $\mathbf{A}$ will be denoted by $\mathbf{A}_{n-\text { row }}$ and $\mathbf{A}_{n-\text { col }}$ respectively. In order to discuss the effect of random error on variance and bias in the estimated eigenvalues, it is necessary to include the random error in the model equations. If the elements of $\mathbf{A}$ are unbiased, $\mathbf{A}$ can be decomposed as $\tilde{\mathbf{A}}+\delta \mathbf{A}$, where the 'tilde' indicates the true values and the matrix $\delta \mathbf{A}$ contains only random error. This notation will be used, for example, for the pure component profiles in equation (1). Biased quantities such as the eigenvalues will not be decomposed this way (see Appendix III).

\section{BIAS AND VARIANCE RESULTING FROM RANDOM ERRORS}

It is important to note that we will deal with bias and variance in the estimated parameters that result from random measurement noise. There is no bias in the data and the factor models used will not be underdimensioned. It will be shown that the estimated parameters are intrinsically biased if the calculation involves a non-linear transformation of the data, which is certainly the case for rank annihilation. For the derivation of variance expressions we will make use of first-order approximations. ${ }^{7}$ For the derivation of bias expressions additional approximations are needed. A systematic numerical evaluation must point out whether these approximations can be justified. Usually the assumption of uncorrelated, homoscedastic noise will be made. However, the assumption of homoscedasticity is only made in order to obtain tractable expressions and these expressions are in fact obtained after simplifying the expressions derived for heteroscedastic noise. The results are distribution-free, since we only use the size of the errors, i.e. we investigate how the standard error of the parent distribution is propagated by the system of equations under study. We will illustrate these points by a simple example that can be worked out by hand, because we assume a special distribution for the errors. A good discussion of the problem can also be found in Reference 8 , where the influence of random error on the result of univariate standard addition is discussed using more realistic distributions.

Assume that we measure two random variables $X$ and $Y$ and we want to estimate the ratio $Z=X / Y$. This problem is extensively treated in the statistics literature. ${ }^{9}$ In our specific example the true values $\tilde{X}$ and $\tilde{Y}$ are 10 and the measurement error can only take two values, -1 and +1 . Therefore the sets of possible outcomes for $X, Y$ and $Z$ are

$$
\begin{gathered}
\Omega_{X}=\Omega_{Y}=\{9,11\} \\
\Omega_{L}=\left\{\frac{9}{9}, \frac{9}{11}, \frac{11}{9}, \frac{11}{11}\right\}
\end{gathered}
$$

The true bias in $Z$ is calculated as the difference between the expected value for $Z$, denoted by $E[Z]$, and the true value for $Z$, i.e. $\tilde{Z}=\tilde{X} / \bar{Y}=1$. The expected value for $Z$ is the mean for the four possible outcomes, $\dot{\dagger}$ i.e. $E[Z]=1 \cdot 0101$. It follows that the estimated ratio is biased

\footnotetext{
* The pure component protiles are also biased, but calculations show that the bian in neglible compared with the variance in the regine where the bias in the eigensalues is already considerable.

+ In general we have many pomible outcomes and we evaluate newly derived expression by llonte (arlo smulation.
} 
upwards and the bias in $Z$, bias $(Z)$, takes the value of +0.0101 here. The predicted value for $E[Z]$ is 1.0100 (see equation (41) in Appendix 1) and the predicted bias is therefore +0.0100 . It is seen that the agreement between predicted and 'experimental' values is excellent. The true variance in $Z$ is given by $\operatorname{var}(Z)=E\left[Z^{2}\right]-(E[Z])^{2}=0 \cdot 0205$, whereas the predicted value is $0 \cdot 0200$ (see equation (42) in Appendix I). Again the agreement is excellent. However, it should be noted that in order to evaluate the predicted value, we used the true values for $X, Y$ and the standard deviation of the parent distribution. If instead we inserted experimental values, we should expect to predict bias and variance with an error that is dictated by the size of the experimental error (about 10\%). We merely used the theoretical values to indicate that the resulting predictions do not depend on distributional assumptions for the noise. The largest errors must be expected to be caused by the evaluation of these expressions using experimental values. ${ }^{10}$

\section{BIAS IN THE EIGENVALUES}

In the preceding section we showed how random measurement noise induces bias in the estimated parameters if the calculation involves a non-linear transformation. Measurement noise enters the rank annihilation procedure at two different places. First, there may be errors in the concentrations. These errors are introduced, for example, by the sample preparation process or the injection volume irreproducibility. This error is independent of the second kind of error, which is caused by the detection process eventually leading to the data matrices for the unknown and calibration sample. Usually the error in the concentration is (much) larger than the error in the measured response and three possibilities for the eigenvalues found by rank annihilation are relevant. Consequently we introduce three different symbols for the eigenvalues.

(1) There are no errors in the concentrations or in the measured response:

$$
\tilde{\boldsymbol{\Pi}}=\tilde{\mathbf{C}}_{\mathrm{M}}^{-1} \tilde{\mathbf{C}}_{\mathrm{N}}
$$

and it is evident that the true concentration ratios must be found.

(2) There are only errors in the concentrations:

$$
\Pi=\mathbf{C}_{\mathrm{M}}^{-1} \mathbf{C}_{\mathrm{N}}
$$

and in this case the concentration ratios found are biased upwards as shown in the preceding section.

(3) There are errors in the concentrations and in the measured response and (3a) becomes

$$
\hat{\Pi}=\mathbf{H}^{+} \mathbf{N} \mathbf{Y}^{\ddagger}
$$

Now, since the pseudoinverse matrices are not free from error, an additional bias is introduced (see Appendix 1). It is important to note that the equivalent expression for multivariate calibration contains the pseudoinverse of a matrix that enters the calculation, whereas in equation $(4 \mathrm{c})$ the pseudoinverse matrices are calculated for the reconstructed profiles. Because of the different independent contributions to the bias, the following is immediate for practical situations:

$$
E[\hat{\mathbf{\Pi}}] \neq E[\mathbf{\Pi}] \neq \tilde{\mathbf{\Pi}}
$$

In the remaining sections we will refer to the eigenvalues defined by equations (4) as true, actual and estimated concentration ratios respectively. The bias in the actual concentration 
ratio is easily estimated from equation (41). The biasing effect of the error in the reconstructed profiles is derived by considering the error in the scores and loadings (Appendix II). We will work out these expressions for Lorber's method and the generalizations of Sánchez and Kowalski and of Wilson et al.

\section{Lorber's method}

First, the model represented by equation (1) is rewritten as

$$
\begin{gathered}
\mathbf{M}=\mathbf{H}_{\mathbf{M}} \mathbf{Y}_{\mathrm{M}}^{\mathrm{T}}=\left(\tilde{\mathbf{H}}_{\mathrm{M}}+\delta \mathbf{H}_{\mathrm{M}}\right)\left(\tilde{\mathbf{Y}}+\delta \mathbf{Y}_{\mathrm{M}}\right)^{\mathrm{T}} \\
\mathbf{N}=\mathbf{H}_{\mathrm{N}} \boldsymbol{\Pi}_{\mathrm{N}} \mathbf{Y}_{\mathrm{N}}^{\mathrm{T}}=\left(\tilde{\mathbf{H}}_{\mathrm{M}}+\delta \mathbf{H}_{\mathrm{N}}\right) \boldsymbol{\Pi}_{\mathrm{N}}\left(\tilde{\mathbf{Y}}+\delta \mathbf{Y}_{\mathrm{N}}\right)^{\mathrm{T}}
\end{gathered}
$$

The subscripts of the experimental profiles, the error terms and the eigenvalue matrix refer to the matrix from which they are derived. The concentration dependence implicit in the column profiles of $\tilde{\mathbf{H}}$ is also indicated by adding a subscript. The resulting prediction equation is

$$
\hat{\boldsymbol{\Pi}}_{\mathbf{N}}=\mathbf{H}_{\mathbf{M}}^{+} \mathbf{N} \mathbf{Y}
$$

The errors in the profiles of $\mathbf{N}$ and $\mathbf{M}$ are uncorrelated. Therefore the expected value of the eigenvalues can be evaluated using equations (46), (56) and (57) as

$$
\begin{aligned}
& E\left[\hat{\Pi}_{\mathrm{N}}\right]=E\left[\mathbf{H}_{\mathrm{M}}^{+} \mathbf{N} \mathbf{Y}_{\mathrm{M}}^{\ddagger}\right] \\
& =E\left[\mathbf{H}_{\mathrm{M}}^{+}\right] E\left[\mathbf{H}_{\mathrm{N}}\right] E\left[\boldsymbol{\Pi}_{\mathrm{N}}\right] E\left[\mathbf{Y}_{\mathrm{N}}^{\mathrm{T}}\right] E\left[\mathbf{Y}_{\mathrm{M}}^{+}\right] \\
& =\left[\mathbf{I}+\left(\tilde{\mathbf{H}}_{\mathbf{M}}^{\mathrm{T}} \tilde{\mathbf{H}}_{\mathbf{M}}\right)^{-1} E\left[\delta \mathbf{H}_{\mathbf{M}}^{\mathrm{T}} \delta \mathbf{H}_{\mathbf{M}}\right]\right]^{-1} E\left[\boldsymbol{\Pi}_{\mathbf{N}}\right]\left[\mathbf{I}+E\left[\delta \mathbf{Y}_{\mathbf{M}}^{\mathrm{T}} \delta \mathbf{Y}_{\mathbf{M}}\right]\left(\tilde{\mathbf{Y}}^{\mathrm{T}} \tilde{\mathbf{Y}}\right)^{-1}\right]^{-1} \\
& =\left(\mathbf{I}+S \sigma_{M}^{2} \tilde{\mathbf{Y}}_{M}\right)^{-1} E\left[\Pi_{N}\right]\left(\mathbf{I}+W \sigma_{M}^{2} \tilde{\mathbf{\Psi}}_{M}\right)^{-1}
\end{aligned}
$$

where the symbol $\boldsymbol{\Psi}=\mathbf{T}^{-1} \boldsymbol{\Lambda}^{-1} \mathbf{T}$ is introduced. If the errors are small, the bias in the estimated eigenvalues can be evaluated by only considering the diagonal elements of the correction terms (see equation (47)). The result is

$$
\operatorname{bias}\left(\hat{\pi}_{\mathrm{N}, n}\right)=E\left[\hat{\pi}_{\mathrm{N}, n}\right]-E\left[\pi_{\mathrm{N}, n}\right]=-(S+W) \sigma_{\mathrm{M}}^{2} \tilde{\Psi}_{\mathrm{M}, n n} E\left[\hat{\pi}_{\mathrm{N}, n}\right]
$$

where $\pi_{\mathrm{N}, n}=\Pi_{\mathrm{N}, n n}$. The bias induced by errors in the response is proportional to the size of the eigenvalue and, since the matrix $\tilde{\mathbf{Y}}_{M}$ is positive definite, is expected to have a negative sign. Analogously to multivariate calibration, we can define $\boldsymbol{\Psi}$ as the variance factor ${ }^{11}$ and again several transcriptions are possible. Summarizing gives

$$
\begin{aligned}
\Psi_{n n} & =\left(\mathbf{H}^{\mathrm{T}} \mathbf{H}\right)_{n n}^{-1}\left(\mathbf{Y}^{\mathrm{T}} \mathbf{Y}\right)_{n n}^{-1} \\
& =\left\|\mathbf{H}_{n-\text { row }}^{+}\right\|^{2}\left\|\mathbf{Y}_{n-\text { col }}^{+}\right\|^{2} \\
& =\sum_{p=1}^{F}\left(\frac{T_{p n}}{\theta_{\mathrm{M}, p}}\right)^{2} \\
& =(\mathrm{SEL}))_{\mathrm{X}, n}^{-2}(\mathrm{SEL})_{\mathrm{Y}, n}^{-2} c_{\mathrm{M}, n}^{-2}
\end{aligned}
$$

where $\|\cdot\|$ is the Euclidean vector norm and $\theta_{\mathrm{M}, p}=\theta_{\mathrm{M}, p p}$. The close relation between multivariate and bilinear calibration is apparent from equations (10a) and (10b). The equivalent for (50c) now contains the elements of the eigenvectors. In essence it shows that in rank annihilation the two fundamental problems of linear algebra, i.e. the least squares problem and the eigenvalue problem, are combined. It is important to note that the equivalent of $(50 \mathrm{~d})$ contains Lorber's selectivities ${ }^{12}$ in the separate modes of the data. The situation is different from the univariate and multivariate case, where we have seen that the error in the 
determined concentration depends on the sensitivity of the measurement. The generalization of the sensitivity concept from multivariate to bilinear calibration does not seem to be straightforward. It should be noted that Ho et al. ${ }^{13}$ have derived an expression for the variance factor for the iterative procedure that is similar to equation (10d). The only difference from the current result is the presence of a factor of two. A comparison shows that their 'uniqueness' $q$ relates to Lorber's selectivity as $q=(\mathrm{SEL})$. The extension to three-way data is straightforward as shown by Appelof and Davidson. ${ }^{2,14}$ However, these theories do not include the error in the calibration matrix, thus limiting their practical usefulness considerably.

\section{Generalization of Sánchez and Kowalski}

In the generalization of Sánchez and Kowalski the matrices $\mathbf{N}$ and $\mathbf{M}$ are replaced by $\mathbf{M}$ and $\mathbf{Q}=\mathbf{N}+\mathbf{M}$ respectively. The analogy of equation (6) is

$$
\begin{gathered}
\mathbf{Q}=\mathbf{H}_{\mathbf{Q}} \mathbf{Y}_{\mathrm{Q}}^{\mathrm{T}}=\left(\tilde{\mathbf{H}}_{\mathbf{Q}}+\delta \mathbf{H}_{\mathrm{Q}}\right)\left(\tilde{\mathbf{Y}}+\delta \mathbf{Y}_{\mathrm{Q}}\right)^{\mathrm{T}} \\
\mathbf{M}=\mathbf{H}_{\mathbf{M}} \boldsymbol{\Pi}_{\mathbf{M}} \mathbf{Y}_{\mathbf{M}}^{\mathrm{T}}=\left(\tilde{\mathbf{H}}_{\mathbf{Q}}+\delta \mathbf{H}_{\mathbf{M}}\right) \boldsymbol{\Pi}_{\mathbf{M}}\left(\tilde{\mathbf{Y}}+\delta \mathbf{Y}_{\mathbf{M}}\right)^{\mathrm{T}} \\
\mathbf{N}=\mathbf{H}_{\mathbf{N}}\left(\mathbf{I}-\mathbf{\Pi}_{\mathbf{M}}\right) \mathbf{Y}_{\mathbf{N}}^{\mathrm{T}}=\left(\tilde{\mathbf{H}}_{\mathbf{Q}}+\delta \mathbf{H}_{\mathbf{N}}\right)\left(\mathbf{I}-\boldsymbol{\Pi}_{\mathbf{M}}\right)\left(\tilde{\mathbf{Y}}+\delta \mathbf{Y}_{\mathbf{N}}\right)^{\mathrm{T}}
\end{gathered}
$$

The analogy of equation (7) is

$$
\hat{\mathbf{\Pi}}_{\mathbf{M}}=\mathbf{H}_{\mathbf{Q}}^{+} \mathbf{M Y} \mathbf{Y}_{\mathbf{Q}}^{\ddagger}
$$

The errors in $\mathbf{M}$ and $\mathbf{Q}$ are correlated and the same holds for the errors in the reconstructed profiles. Consequently, cross-terms containing the covariance matrix $E\left[\delta \mathbf{H}_{Q}^{\top} \delta \mathbf{H}_{M}\right]$ will arise in the evaluation of the expectation of the eigenvalues:

$$
\begin{aligned}
E\left[\hat{\mathbf{\Pi}}_{\mathrm{M}}\right]= & E\left[\mathbf{H}_{\mathrm{Q}}^{+} \mathbf{M Y} \mathbf{Y}^{\dagger}\right] \\
& E\left[\mathbf{H}_{\mathrm{Q}}^{+} \mathbf{H}_{\mathrm{M}}\right] E\left[\boldsymbol{\Pi}_{\mathrm{M}}\right] E\left[\mathbf{Y}_{\mathrm{M}}^{\mathrm{T}} \mathbf{Y}_{\mathrm{Q}}^{\ddagger}\right]
\end{aligned}
$$

The problem of evaluating the covariance matrix is solved by noting that to first order an element of $\mathbf{H}_{\mathrm{Q}}$ is given by

$$
H_{\mathrm{Q}, i p}=H_{\mathrm{M}, i p} \pi_{\mathrm{M}, p}+H_{\mathrm{N}, i p}\left(1-\pi_{\mathrm{M}, p}\right)
$$

and the errors in the profiles are related as

$$
\delta \mathbf{H}_{\mathrm{M}}=\delta \mathbf{H}_{\mathrm{Q}} \boldsymbol{\Pi}_{\mathrm{M}}^{-1}
$$

Working out equation (13) eventually results in

$$
E\left[\hat{\Pi}_{\mathrm{M}}\right]=\left(\mathbf{I}+S \sigma_{\mathrm{Q}}^{2} \tilde{\mathbf{\Psi}}_{\mathrm{Q}}\right)^{-1}\left(\mathbf{I}+S \sigma_{\mathrm{M}}^{2} \tilde{\mathbf{\Psi}}_{\mathrm{Q}} \boldsymbol{\Pi}_{\mathrm{M}}^{-1}\right) E\left[\boldsymbol{\Pi}_{\mathrm{M}}\right]\left(\mathbf{I}+W \sigma_{\mathrm{M}}^{2} \hat{\boldsymbol{\Pi}}_{\mathrm{M}}^{-1} \tilde{\mathbf{\Psi}}_{\mathrm{Q}}\right)\left(\mathbf{I}+W \sigma_{\mathrm{Q}}^{2} \tilde{\mathbf{\Psi}}_{\mathrm{Q}}\right)^{-1}
$$

It is seen that two cross-terms are introduced that have a cancelling effect. In fact, this observation is somewhat misleading, as follows from the analogy of equation (9),

$$
\operatorname{bias}\left(\hat{\pi}_{\mathrm{M}, n}\right)=\left[-(S+W) \sigma_{\mathrm{Q}}^{2}+(S+W-2 F-2) \sigma_{\mathrm{M} \pi}^{2} \pi_{\mathrm{M}, n}^{-1}\right] \tilde{\Psi}_{\mathrm{Q}, n n} E\left[\hat{\pi}_{\mathrm{M}, n}\right]
$$

If the response error is identical for $\mathbf{N}$ and $\mathbf{M}$ and $F \ll \min (S, W)$, there will be almost no bias if the concentrations are identical for both samples. The situation is also quite favorable if the concentrations are of the same order of magnitude. However, for components that have a low concentration in the unknown sample, a large bias may be found compared with the previous case. (It should be noted that in order to compare equations (9) and (17), the normalization of the profiles in $\mathbf{H}$ must be taken into account.) 


\section{Generalization of Wilson et al.}

In the generalization of Wilson et al. the matrices $\mathbf{N}$ and $\mathbf{M}$ are adjoined rather than added in order to define the factor space. The column space is found by decomposing the column augmented matrix $\mathbf{Q}_{\mathrm{c}}$ as

$$
\mathbf{Q}_{\mathrm{C}}=(\mathbf{N} \mid \mathbf{M})=\mathbf{U}_{\mathrm{C}} \boldsymbol{\theta}_{\mathrm{C}} \mathbf{V}_{\mathrm{C}}^{\mathrm{T}}
$$

and the row space is found by decomposing the row augmented matrix $\mathbf{Q}_{\mathrm{R}}$ as

$$
\mathbf{Q}_{\mathrm{R}}=\left(\frac{\mathbf{N}}{\mathbf{M}}\right)=\mathbf{U}_{\mathrm{R}} \boldsymbol{\theta}_{\mathrm{R}} \mathbf{V}_{\mathrm{R}}^{\mathrm{T}}
$$

We have chosen to decompose the matrices using the SVD in order to parallel the preceding discussion. In fact, any orthogonal decomposition should give identical results. The matrices $\mathbf{N}$ and $\mathbf{M}$ are now denoted as in equation (6) and the resulting eigenvalue problem is (for more details see Reference 5)

$$
\mathbf{N}_{U v} \mathbf{Z}_{\mathrm{V}}=\mathbf{M}_{\mathrm{Uv}} \mathbf{Z}_{\mathrm{V}} \hat{\Pi}_{\mathrm{N}}
$$

where $\mathbf{N}_{U v}=\mathbf{U}_{\mathrm{C}}^{\mathrm{T}} \mathbf{N} \mathbf{V}_{\mathbf{R}}$ and $\mathbf{M}_{\mathbf{U v}}=\mathbf{U}_{\mathrm{C}}^{\mathrm{T}} \mathbf{M} \mathbf{V}_{\mathbf{R}}$. Equation (19) can be converted into the analogy of equations (7) and (12) by introducing the following expression for the reconstructed profiles:

$$
\begin{gathered}
\mathbf{H}_{\mathrm{C}}=\mathbf{U}_{\mathrm{C}} \mathbf{M}_{\mathrm{UV}} \mathbf{Z}_{\mathrm{V}} \\
\mathbf{Y}_{\mathrm{R}}^{\mathbf{T}}=\mathbf{Z}_{\mathrm{V}}^{-1} \mathbf{V}_{\mathrm{R}}^{\mathbf{T}}
\end{gathered}
$$

Again cross-terms arise, now containing $E\left[\delta \mathbf{H}_{\mathrm{C}}^{\mathrm{T}} \delta \mathbf{H}_{\mathrm{N}}\right]$, which can be worked out by taking the correlations into account. First it is recognized that errors in $\mathbf{H}_{\mathbf{N}}$ are related to errors in $\mathbf{N}$ as follows:

$$
\begin{aligned}
\delta H_{N} & =\delta N Y Y_{N} \Pi_{N}^{-1} \\
& \approx \delta N Y Y_{\mathrm{N}}^{-1} \\
& =\delta N V_{R} Z_{V} \Pi_{N}^{-1}
\end{aligned}
$$

Next $\mathbf{H}_{C}$ is rewritten as

$$
\mathbf{H}_{\mathrm{C}}=\mathbf{U}_{\mathrm{C}} \boldsymbol{\theta}_{\mathrm{C}} \mathbf{T}_{\mathrm{C}}
$$

and the errors in $\mathbf{H}_{\mathrm{C}}$ are related to the errors in $\mathbf{Q}_{C}$ using the method described in Appendix II. Finally the covariance matrix is evaluated by correlating the errors in $\mathbf{N}$ and the corresponding part of $\mathbf{Q}_{c}$. The same procedure is followed for the row profiles. The resulting expression is

$$
\begin{aligned}
E\left[\hat{\Pi}_{\mathrm{N}}\right] & =\left[\mathbf{I}+\operatorname{So}_{\mathrm{N}}^{2}\left(\mathbf{T}_{\mathrm{C}}^{-1} \boldsymbol{\Lambda}_{\mathrm{C}}^{-1} \mathbf{T}_{\mathrm{C}}\right)\right]^{-1}\left(\mathbf{I}+\frac{S}{2} \sigma_{\mathrm{N}}^{2}\left(\mathbf{T}_{\mathrm{C}}^{-1} \boldsymbol{\Lambda}_{\mathrm{C}}^{-1} \mathbf{V}_{\mathrm{C}}^{\mathrm{T}} * \mathbf{V}_{\mathrm{R}} \mathbf{T}_{\mathrm{R}} \Pi_{\mathrm{N}}\right)\right) E\left[\boldsymbol{\Pi}_{\mathrm{N}}\right] \\
& =\left(\mathbf{I}+\frac{W}{2} \sigma_{\mathrm{N}}^{2}\left(\boldsymbol{\Pi}_{\mathrm{N}} \mathbf{T}_{\mathrm{C}}^{-1} \boldsymbol{\theta}_{\mathrm{C}}^{-1} \mathbf{U}_{\mathrm{C}}^{\mathbf{T}} * \mathbf{U}_{\mathrm{R}} \boldsymbol{\theta}_{\mathrm{R}}^{-1} \mathbf{T}_{\mathrm{R}}\right)\right)\left[\mathbf{I}+W \sigma_{\mathrm{N}}^{2}\left(\mathbf{T}_{\mathrm{R}}^{-1} \boldsymbol{\Lambda}_{\mathrm{R}}^{-1} \mathbf{T}_{\mathrm{R}}\right)\right]^{-1}
\end{aligned}
$$

where the substitution $\mathbf{T}_{\mathbf{R}}=\mathbf{Z}_{\mathrm{V}}$ is made for simplifying reasons and the ' $*$ ' indicates that the inner products are taken over the admissible range, since the matrices involved are not conformable. It is seen that the contributions of the column and row profiles are not symmetrical for the cross-terms. There is also an important point about the practical evaluation of equation (23). The matrices $\mathbf{T}_{\mathbf{R}}$ and $\mathbf{T}_{C}$ must be normalized in the same way to 
fix the value of these cross-terms. It is tempting to speculate on this undesirable behavior: we think that this untractable expression arises because the relation between the errors in the reconstructed column and row responses is destroyed, since they result from the decomposition of two different matrices (compare equations (56) and (57)). We have not found a way of simplifying this result (note the presence of $\boldsymbol{\theta}_{\mathrm{C}}$ and $\boldsymbol{\theta}_{\mathrm{R}}$ in one term) and consequently we have no analogy for equations (9) and (17).

\section{VARIANCE IN THE EIGENVALUES}

In a previous paper ${ }^{7}$ we derived variance expressions by performing error propagation on the standard eigenvalue problem of Lorber, i.e. equation (3d). This procedure leads to standard errors expressed in the abstract decomposition of $\mathbf{M}$. In this paper we will derive standard errors expressed in the physical decomposition of $\mathbf{M}$ by performing error propagation on equation (7). The derivation (see Appendix III) is much shorter and the assumptions and approximations made are more transparent.

\section{Lorber's method}

Using the notation developed in the preceding section, equation (62) is rewritten as

$$
\operatorname{var}\left(\hat{\pi}_{N, n}\right)=\tilde{\Psi}_{M, n n}\left(\sigma_{N}^{2}+\pi_{N, n}^{2} \sigma_{M}^{2}\right)
$$

This expression can be combined with equation (9) to give the mean squared error (MSE) as in equation (49).

\section{Generalization of Sánchez and Kowalski}

Taking the correlations between the elements of $\mathbf{M}$ and $\mathbf{M}+\mathbf{N}$ into account gives

$$
\operatorname{var}\left(\hat{\pi}_{\mathrm{M}, n}\right)=\tilde{\Psi}_{\mathrm{Q}, n n}\left(\sigma_{\mathrm{M}}^{2}-2 \pi_{\mathrm{M}, n} \sigma_{\mathrm{M}}^{2}+\pi_{\mathrm{M}, n}^{2} \sigma_{\mathrm{Q}}^{2}\right)
$$

This expression can be combined with equation (17) to give the MSE. Comparing equations (24) and (25), one finds that the variance is always reduced. This is an important result but difficult to prove for more general cases, i.e. heteroscedastic or correlated measurement noise. We will show in a later section (Variance in the unknown concentrations) that this variance reduction must be found in general.

\section{Generalization of Wilson et al.}

The variance expression for the generalization of Wilson et al. is easily derived by noting that in fact the standard eigenvalue problem of Lorber is solved if the unknown data matrix spans the factor space. There is, however, a difference in the evaluation of equation (24), since the pure quantities have to be replaced by the experimental values. Since the profiles are now reconstructed using information from both matrices, they are expected to be more precise. Therefore one should estimate the same variance but the estimate itself may be more precise.

\section{VARIANCE IN THE BIAS-CORRECTED EIGENVALUES}

Least squares estimators are characterized by their low variance. However, with errors in the regression matrix they are no longer unbiased. ${ }^{6}$ Taking the structure of the errors in the 
regression matrix into account, so-called measurement error models can be built that have a reduced MSE. ${ }^{15}$ For rank annihilation we also have an errors-in-variables situation. The important question is therefore: is bias negligible under practical circumstances or (if this is not the case) can we construct better estimators? Before turning to complicated measurement error models (which is far beyond the scope of this paper), we will show that if the bias estimate is adequate, we can reduce the MSE by simply correcting for the bias. We will work out the principle for Lorber's method and the generalization of Sánchez and Kowalski. Assuming that $F \ll \min (S, W)$, dropping the subscripts for simplicity and replacing the true quantities by their experimental counterparts, equations (9) and (17) can be written as

$$
\begin{gathered}
\operatorname{biâs}(\hat{\pi})=\varepsilon \hat{\pi} \\
\operatorname{biâs}(\hat{\pi})=\varepsilon(1-2 \hat{\pi})
\end{gathered}
$$

leading to the (almost) unbiased estimators

$$
\begin{gathered}
\hat{\pi}(\varepsilon)=\hat{\pi}-\varepsilon \hat{\pi} \\
\hat{\pi}(\varepsilon)=\hat{\pi}+2 \varepsilon \hat{\pi}-\varepsilon
\end{gathered}
$$

which have an approximate MŜE given by

$$
\begin{gathered}
\operatorname{MSEE}(\hat{\pi}(\varepsilon))=\left(1+\varepsilon^{2}\right) \operatorname{vâr}(\hat{\pi})+(\hat{\pi})^{2} \operatorname{vâr}(\varepsilon) \\
\operatorname{MSE}(\hat{\pi}(\varepsilon))=\left(1+4 \varepsilon^{2}\right) \operatorname{vâr}(\hat{\pi})+\left[1+4(\hat{\pi})^{2}\right] \operatorname{vâr}(\varepsilon)
\end{gathered}
$$

These values have to be compared with the MŜE for the uncorrected estimator. We will illustrate the possible beneficial effect of the proposed bias correction for the generalization of Sánchez and Kowalski. Assume that $\hat{\pi}=0 \cdot 1$, vâr $(\hat{\pi})=10^{-4}$ and biâs $(\hat{\pi})=10^{-2}$. In this specific example the standard error and bias are equal $(10 \%)$. Furthermore, the relative standard error in the estimated bias is assumed to be $10 \%$. Working out $(28 \mathrm{~b})$ shows that $\operatorname{MSE}(\hat{\pi}(\varepsilon)) \approx 10^{-4}$ compared with $\operatorname{MSE}(\hat{\pi})=\sqrt{2} \times 10^{-4}$. The bias correction works very well in this specific example. However, it is important to note that the error in the estimated bias is an unknown quantity and the method should only be applied if it is reasonable to assume that this error is not underestimated.

\section{CONFIDENCE LIMITS AND LIMITS OF DETECTION FOR THE ACTUAL CONCENTRATION RATIOS}

The Monte Carlo simulations described later show that the sampling distribution of the estimated eigenvalues is approximately Gaussian. (This conclusion was arrived at by investigating percentiles and higher moments.) This means that the error estimates derived in the preceding sections can be used to construct confidence intervals for these eigenvalues in the usual way. It is, however, more interesting to have the confidence intervals for the actual concentration ratios. These intervals cannot be found by simply inverting the intervals for the estimated eigenvalues, since the errors depend on the corresponding eigenvalues. This problem is solved by transforming the eigenvalues in such a way that the errors become independent of the eigenvalues. ${ }^{16}$ We will illustrate the necessary steps for the bias-corrected eigenvalues and restrict ourself to Lorber's method. We assume that the estimate for the bias is accurate. Then neglecting the last term in equation (28a) gives

$$
\operatorname{vâr}(\hat{\pi}(\varepsilon))=\operatorname{MSEE}(\hat{\pi}(\varepsilon)) \cong\left(1+\varepsilon^{2}\right) \Psi_{n n} \hat{\sigma}_{M}^{2}\left[1+\hat{\pi}(\varepsilon)^{2}\right]
$$


A transformation $h$ is needed that stabilizes the variance ${ }^{16}$

$$
\operatorname{var}(h(\hat{\pi}(\varepsilon)))=\left[h^{\prime}(\hat{\pi}(\varepsilon))\right]^{2} \operatorname{var}(\hat{\pi}(\varepsilon)) \equiv c
$$

This transformation is found to be

$$
h(\hat{\pi}(\varepsilon))=\frac{\ln \left[\hat{\pi}(\varepsilon)+\sqrt{ }\left(\hat{\pi}(\varepsilon)^{2}+1\right)\right]}{\left(1+\varepsilon^{2}\right) \Psi_{n n}^{1 / 2} \hat{\sigma}_{M}}
$$

Smooth transformations of a normally distributed random variable are also approximately normally distributed, so: ${ }^{16}$

$$
\mathscr{L}\{h(\hat{\pi}(\varepsilon))-h(\pi)\}=N(0,1)
$$

The interval for the transformed variable with confidence $1-\alpha$ is given by

$$
h(\hat{\pi}(\varepsilon))-z(\alpha) \leqslant h(\pi) \leqslant h(\hat{\pi}(\varepsilon))+z(\alpha)
$$

and the confidence interval for the actual concentration ratio is found by back-transformation. It must be kept in mind that the terms contributing to the far right-hand side of equation (29) are not fixed. The confidence intervals derived are therefore only approximate. They may, however, be useful in practice, since no exact analytical results have been reported until now.

Since the detection of a component depends exclusively on the size of the eigenvalue, the detection limit for rank annihilation should be based on the standard error in the eigenvalue. Detection limits have been derived for multivariate calibration by Lorber ${ }^{12}$ for the homoscedastic case and by Bauer et al. ${ }^{17}$ for the heteroscedastic case. The methods proposed by these authors can be duplicated for rank annihilation, since the relevant expressions are very similar. However, if bias is important, it should be taken into account.

\section{BIAS IN THE DETERMINED CONCENTRATIONS}

For Lorber's method and the generalization of Wilson et al. we have, after inserting the relevant quantities in equation (41),

$$
\frac{\operatorname{bias}\left(\hat{c}_{\mathrm{M}, n}\right)}{\tilde{c}_{\mathrm{M}, n}}=\left(\frac{\sigma_{\tilde{\pi}_{\mathrm{N}, n}}}{\pi_{\mathrm{N}, n}}\right)^{2}
$$

where $c_{\mathrm{M}, n}=C_{\mathrm{M}, n n}$. For the generalization of Sánchez and Kowalski we have to introduce a covariance term. ${ }^{8}$ The result is

$$
\frac{\operatorname{bias}\left(\hat{c}_{\mathrm{M}, n}\right)}{\tilde{c}_{\mathrm{M}, n}}=2\left(\frac{\sigma_{\hat{\pi}_{\mathrm{M}, n}}}{1-\pi_{\mathrm{M}, n}}\right)^{2}
$$

It follows that the bias is reduced if $c_{N, n}>(\sqrt{2}-1) c_{M, n}$. We have seen, however, that bias can be corrected to a certain extent and this result is therefore only important if the bias correction does not work.

\section{VARIANCE IN THE UNKNOWN CONCENTRATIONS}

By performing simple error propagation on the ratio of numbers and inserting the result for the eigenvalues, we find for the variance in the unknown concentration for Lorber's method 
and the generalization of Wilson et al.

$$
\begin{aligned}
\operatorname{var}\left(\hat{c}_{\mathrm{M}, n}\right) & =\tilde{c}_{\mathrm{M}, n}^{2}\left[\left(\frac{\sigma_{\hat{\pi}_{\mathrm{N}, n}}}{\pi_{\mathrm{N}, n}}\right)^{2}+\left(\frac{\sigma_{\hat{C}_{\mathrm{N}, n}}}{\tilde{c}_{\mathrm{N}, n}}\right)^{2}\right] \\
& =\tilde{c}_{\mathrm{M}, n}^{2}\left[(\mathrm{SE} \mathrm{L}) \overline{\mathrm{x}}_{, n}^{2}(\mathrm{SE} \mathrm{L}) \overline{\mathrm{Y}}_{, n}^{2}\left[\left(\frac{\sigma_{\mathrm{N}}}{\tilde{c}_{\mathrm{N}, n}}\right)^{2}+\left(\frac{\sigma_{\mathrm{M}}}{\tilde{c}_{\mathrm{M}, n}}\right)^{2}\right]+\left(\frac{\sigma_{\hat{c}_{\mathrm{N}, n}}}{\tilde{c}_{\mathrm{N}, n}}\right)^{2}\right]
\end{aligned}
$$

Equation (36) shows explicitly the dependence of the estimated error on the expected error sources: the error in the concentration of the calibration sample and the error in the response matrices. The dependence of the error on the value of the unknown concentration reveals the extrapolating character of calibration with only one calibration sample. For the generalization of Sánchez and Kowalski one finds that the contribution of the standard error in the eigenvalues is composed of two parts:

$$
\operatorname{var}\left(\hat{c}_{\mathrm{M}, n}\right)=\tilde{c}_{\mathrm{M}, n}^{2}\left[\left(\frac{1}{1-\pi_{\mathrm{M}, n}}\right)^{2}\left(\frac{\sigma_{\hat{\pi}_{\mathrm{M}, n}}}{\pi_{\mathrm{M}, n}}\right)^{2}+\left(\frac{\sigma_{\hat{\mathcal{C}}_{\mathrm{N}, n}}}{\tilde{c}_{\mathrm{N}, n}}\right)^{2}\right]
$$

The variance in the unknown concentration must, however, remain the same, since we have not added information to the data. It follows that the relative standard error in the eigenvalues is reduced by an amount

$$
\left(1-\pi_{\mathrm{M}, n}\right)^{2} \cong\left(\frac{\tilde{c}_{\mathrm{N}, n}}{\tilde{c}_{\mathrm{N}, n}+\tilde{c}_{\mathrm{M}, n}}\right)^{2}
$$

Since no assumptions were made on the source of the standard error in the eigenvalues, this variance reduction should be found in general, i.e. also for heteroscedastic and correlated noise.

\section{SIMULATIONS}

We simulated three-component systems by multiplying Gaussian elution profiles by experimentally obtained UV spectra for myoglobin (M), $\alpha$-chymotrypsin $(\alpha-C)$ and carbon anhydrase (C). ${ }^{18}$ The spectra and elution profiles are shown in Figures 1 and 2 respectively. The spectra were normalized to unit length in order to make the variance of the individual components proportional to the square of the peak height. The peak positions $(20,25$ and 30$)$ and standard deviations of the peaks $(10,10$ and 10) are chosen in such a way that the chromatographic resolution, defined as $R_{i j}=\left(t_{j}-t_{i}\right) /\left[\left(w_{1 / 2}\right)_{i}+\left(w_{1 / 2}\right)_{j}\right]$, has a value of approximately $0 \cdot 1$. The normalized inner products and correlation coefficients are given in Table 1. These similarity measures are to be compared with the numbers obtained in the recognition step of the rank annihilation procedure. It is seen that although the 'chromatography' is very bad, the overlap of the spectra is much larger. The numbers in Table 1 can, however, not be used in general for obtaining an impression about multicollinearities and therefore we have additionally compiled Lorber's selectivities in Table 2 , since these quantities are directly related to the errors in the eigenvalues. It is seen that the selectivity of the elution profile of $\alpha-\mathrm{C}$ is only twice as large as the selectivity for the spectral mode. This is in sharp contrast with the numbers in Table 1. We added Gaussian noise with a constant standard deviation of $0.05 \mathrm{mAU}$ to both data matrices, i.e. $\mathbf{N}$ and $\mathbf{M}$. This value corresponds to the average residual standard deviation found for three commercially available diode array detectors (HP1040A, PU4021 and Waters 990). This combination of overlap and signal-to-noise ratio is comparable with the most difficult level chosen in Reference 


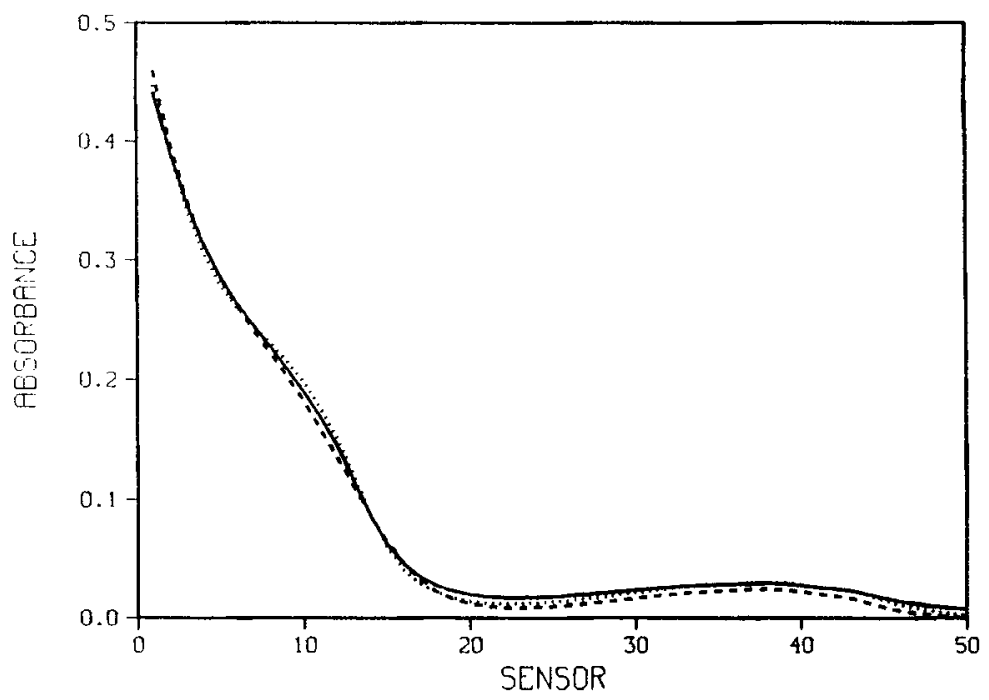

Figure 1. Normalized UV spectra for myoglobine $(\longrightarrow), \alpha$-chymotrypsin $(-\longrightarrow)$ and carbon anhydrase (...)

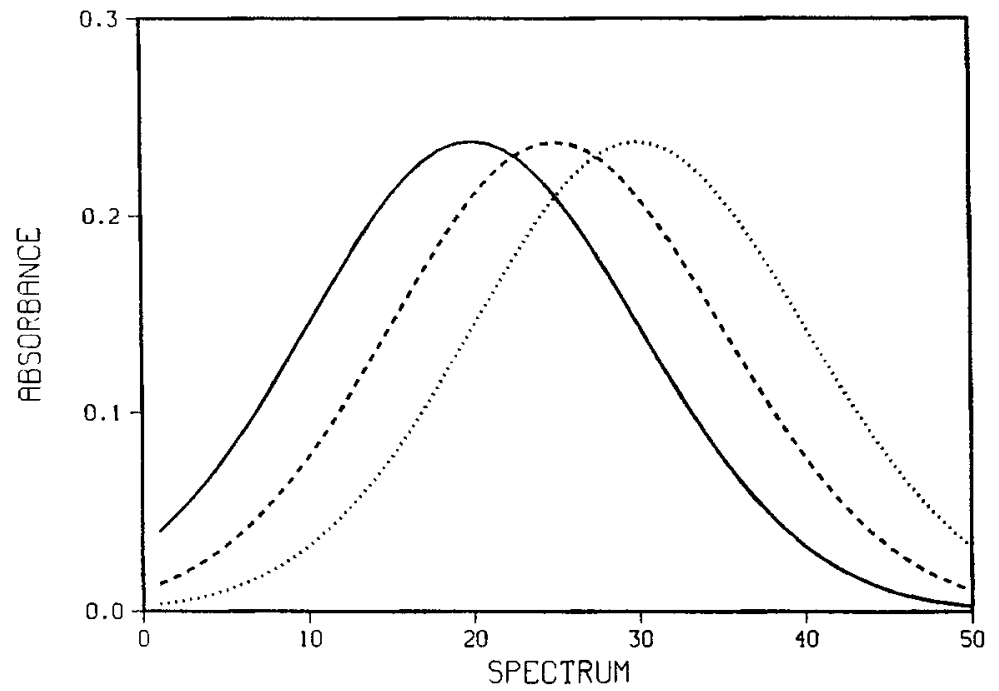

Figure 2. Normalized simulated elution profiles for myoglobine $(-(-), \alpha$-chymotrypsin $(--\longrightarrow)$ and carbon anhydrase $(. .$.

18. There the largest chromatographic overlap corresponded to a value $R_{i j}=0 \cdot 25$ and the standard deviation for the noise was chosen as $0.12 \mathrm{mAU}$. This level proved to be very difficult for curve resolution with iterative target-testing factor analysis (ITTFA). The simulation parameters are summarized in Table 3. 
Table 1. Inner product (right upper corner) and linear correlation coefficient (left lower corner) for UV spectra and elution profiles. Spectra and elution profiles are normalized

\begin{tabular}{|c|c|c|c|c|c|c|}
\hline \multirow[b]{2}{*}{ Standard } & \multicolumn{3}{|c|}{ UV spectrum } & \multicolumn{3}{|c|}{ Elution profile } \\
\hline & $\mathbf{M}$ & $\alpha-\mathrm{C}$ & C & $\mathbf{M}$ & $\alpha-\mathrm{C}$ & $\mathrm{C}$ \\
\hline $\mathrm{M}$ & 1 & 0.9988 & 0.9996 & 1 & 0.94 & 0.78 \\
\hline$\alpha-\mathrm{C}$ & 0.9996 & 1 & 0.9988 & 0.81 & 1 & 0.94 \\
\hline $\mathrm{C}$ & 0.9995 & 0.9989 & 1 & $0 \cdot 32$ & $0 \cdot 81$ & 1 \\
\hline
\end{tabular}

Table 2. Selectivities of UV spectra and elution profiles

\begin{tabular}{lcc}
\hline Standard & UV spectrum & Elution profile \\
\hline $\mathrm{M}$ & 0.0282 & $0 \cdot 1577$ \\
$\alpha-\mathrm{C}$ & 0.0465 & $0 \cdot 0861$ \\
$\mathrm{C}$ & 0.0282 & $0 \cdot 1580$ \\
\hline
\end{tabular}

Table 3. Simulation parameters

\begin{tabular}{lccc}
\hline & M & $\alpha-\mathrm{C}$ & $\mathrm{C}$ \\
\hline Peak position & 20 & 25 & 30 \\
Standard deviation peak & 10 & 10 & 10 \\
Number of spectra & & 50 & \\
Number of wavelengths & & 50 & \\
$\sigma_{\mathrm{N}}=\sigma_{\mathrm{M}}(\mathrm{mAU})$ & & 0.05 & \\
\hline
\end{tabular}

\section{RESULTS AND DISCUSSION}

This section will be divided into two parts. In the first part we will compare the bias and variance for the different models, i.e. Lorber's method, the generalization of Sanchez and Kowalski and the generalization of Wilson et al. It is important to realize that even for data that can be analysed by all methods, the results may be entirely different. In the second part we will study the effect of the dimensionality of the factor space on the errors in the eigenvalues.

\section{Comparison of error estimates for different models}

The developed error estimates are tested by constructing a worst case example: a large spectral and chromatographic overlap is combined with the presence of a diluted component. In Table 4 we give the peak heights encountered during these simulations. $M$ and $C$ are held constant while the peak of $\alpha-\mathrm{C}$ is lowered from 200 to $5 \mathrm{mAU}$. Since both samples contain the same components, rank annihilation should work with the factor space of $\mathbf{N}, \mathbf{M}, \mathbf{N}+\mathbf{M}$ and 
Table 4. Peak heights in mAU for standard and unknown sample

\begin{tabular}{lccc}
\hline Sample & M & $\alpha$-C & C \\
\hline Standard & 300 & 300 & 300 \\
Unknown & 100 & $200-5$ & 300 \\
\hline
\end{tabular}

the adjoined matrices respectively. The analysis with the factor space of $\mathbf{M}$, however, already gives bad results for the second dilution, since the third factor is spoiled by a large embedded error. Therefore we will only give the results for the other decompositions. We will restrict the discussion to the results for the diluted component, since the results obtained for the highest dilution are representative of the results obtained for the major components at all dilutions.

Table 5 summarizes the quantitative solution using the factor space of $\mathbf{N}$, i.e. Lorber's method (reversed). The first two columns give the theoretical values for peak height and eigenvalue. The next four columns give the estimates for the eigenvalue, bias and standard error based on one simulation. The last three columns give the expected values for these quantities based on averaging the eigenvalues obtained for $10^{4}$ simulations. Using $10^{4}$ simulations gives a sample mean for the eigenvalues from which the bias can be accurately estimated. Since we only add noise to the data matrices, the bias is calculated with respect to the true value. (The effect of noise in the concentrations is trivial.) All error estimates are divided by the true value in order to make them comparable. It is seen that the highest dilution gives predicted results for bias and variance that are in excellent agreement with the 'experimental' results. The simple error theory we propose seems to work very well for major components. For the other cases one finds a better agreement for the standard errors than for the bias. This fact can be explained by the additional approximation needed to obtain comprehensive expressions for the bias. Since the contribution of the bias to the MSE is very small, this is of minor importance. Considering only the diagonal elements of the bias correction seems to have a marginal effect on the outcome in all cases. The detection limit is indicated by the standard error to be very near $\tilde{\pi}=0.033$. Since rank annihilation can give a good qualitative solution if there is only one component absent, the predicted standard error

Table 5. Summary of quantitative solution for diluted component: decomposition of $\mathbf{N}$. The numbers printed bold indicate the limit of detection

\begin{tabular}{|c|c|c|c|c|c|c|c|c|}
\hline \multirow[b]{2}{*}{$\tilde{H}$} & \multirow[b]{2}{*}{$\tilde{\pi}$} & \multicolumn{4}{|c|}{ One simulation } & \multicolumn{3}{|c|}{$10^{4}$ simulations } \\
\hline & & $\hat{\pi}$ & $\begin{array}{l}6 / \tilde{\pi}^{\mathrm{a}} \\
(\%)\end{array}$ & $\begin{array}{l}\hat{b} / \tilde{\pi}^{\mathrm{b}} \\
(\%)\end{array}$ & $\begin{array}{l}\hat{\sigma} / \tilde{\pi}^{\mathrm{c}} \\
(\%)\end{array}$ & $E[\tilde{\pi}]$ & $\begin{array}{c}E[b] / \tilde{\pi} \\
(\%)\end{array}$ & $\begin{array}{c}E[\sigma] / \bar{\pi} \\
(\%)\end{array}$ \\
\hline 200 & 0.667 & 0.641 & $-1 \cdot 42$ & $-1 \cdot 42$ & $1 \cdot 79$ & 0.657 & $-1 \cdot 41$ & $1 \cdot 78$ \\
\hline 30 & $0 \cdot 100$ & 0.096 & $-1 \cdot 42$ & $-1 \cdot 40$ & 9.93 & 0.099 & $-1 \cdot 32$ & $9 \cdot 92$ \\
\hline 20 & 0.067 & 0.045 & $-1 \cdot 09$ & $-1 \cdot 06$ & $15 \cdot 7$ & 0.066 & $-1 \cdot 54$ & $14 \cdot 7$ \\
\hline 10 & 0.033 & 0.039 & $-1 \cdot 77$ & $-1 \cdot 71$ & $29 \cdot 1$ & 0.033 & $-2 \cdot 01$ & $29 \cdot 5$ \\
\hline 5 & 0.017 & -0.001 & $+0 \cdot 81$ & +0.93 & $64 \cdot 2$ & 0.016 & $-1 \cdot 49$ & $58 \cdot 3$ \\
\hline
\end{tabular}

${ }^{a}$ Estimated by equation (8).

${ }^{\mathrm{b}}$ Estimated by equation (9).

'Estimated by equation (24). 
is correct, since the influence of the eigenvalue itself is negligible. For the lowest dilution a negative concentration is found. This explains why the predicted bias has changed sign. We were able to locate the detection limit using the predicted standard error. An alternative is to perform target testing on the unknown data matrix. Each target-testing procedure has its own limit of detection and it would be interesting to see whether the results are consistent. It is evident that future research should be focused on reliable quantitation in the neighbourhood of the detection limit.

Table 6 summarizes the quantitative solution using the factor space of $\mathbf{N}+\mathbf{M}$, i.e. the generalization of Sánchez and Kowalski. First we notice that again the agreement is excellent for the first row. Both variance and bias are reduced substantially with respect to the previous case. For the other dilutions we find a variance reduction that is unimportant compared with the increase in bias.

Table 7 summarizes the quantitative solution using the factor space of the adjoined matrices, i.e. the generalization of Wilson et al. First we notice that the agreement between predicted and measured errors is generally not so good as in the previous cases. This is probably caused by the larger contribution of the eigenvalue to the error estimates. The standard error in the

Table 6. Summary of quantitative solution for diluted component: decomposition of $\mathbf{N}+\mathbf{M}$. The numbers printed bold indicate the limit of detection

\begin{tabular}{|c|c|c|c|c|c|c|c|c|}
\hline \multirow[b]{2}{*}{$\tilde{H}$} & \multirow[b]{2}{*}{$\tilde{\pi}$} & \multicolumn{4}{|c|}{ One simulation } & \multicolumn{3}{|c|}{$10^{4}$ simulations } \\
\hline & & $\hat{\pi}$ & $\begin{array}{l}\bar{b} / \tilde{\pi}^{\mathrm{a}} \\
(\%)\end{array}$ & $\begin{array}{l}\hat{b} / \tilde{\pi}^{\mathrm{b}} \\
(\%)\end{array}$ & $\begin{array}{l}\hat{\sigma} / \tilde{\pi}^{\mathbf{c}} \\
(\%))\end{array}$ & $E[\pi]$ & $\begin{array}{c}E[b] / \tilde{\pi} \\
(\%) \mathbf{T}\end{array}$ & $\begin{array}{c}E[\sigma] / \tilde{\pi} \\
(\% 0)\end{array}$ \\
\hline 200 & $0 \cdot 400$ & 0.396 & +0.28 & $+0 \cdot 28$ & $1 \cdot 10$ & $0 \cdot 401$ & +0.24 & $1 \cdot 07$ \\
\hline 30 & 0.091 & 0.098 & $+8 \cdot 35$ & $+8 \cdot 34$ & $8 \cdot 57$ & 0.099 & +9.06 & $8 \cdot 53$ \\
\hline 20 & 0.063 & 0.056 & $+14 \cdot 8$ & $+14 \cdot 8$ & $14 \cdot 5$ & 0.072 & $+14 \cdot 7$ & $13 \cdot 1$ \\
\hline 10 & 0.032 & $0 \cdot 051$ & $+26 \cdot 2$ & $+26 \cdot 2$ & $26 \cdot 2$ & $0 \cdot 043$ & $+31 \cdot 8$ & $27 \cdot 1$ \\
\hline 5 & 0.016 & 0.003 & $+15 \cdot 9$ & $+15 \cdot 9$ & $63 \cdot 8$ & 0.028 & $+67 \cdot 8$ & $55 \cdot 2$ \\
\hline
\end{tabular}

${ }^{a}$ Estimated by equation (16).

${ }^{b}$ Estimated by equation (17).

'Estimated by equation (25).

Table 7. Summary of quantitative solution for diluted component: decomposition of $(\mathbf{N} \mid \mathbf{M})$ and (⿳亠丷⿵冂丶 $)$. The numbers printed bold indicate the limit of detection

\begin{tabular}{|c|c|c|c|c|c|c|c|}
\hline \multirow[b]{2}{*}{$\bar{H}$} & \multirow[b]{2}{*}{$\tilde{\pi}$} & \multicolumn{3}{|c|}{ One simulation } & \multicolumn{3}{|c|}{$10^{4}$ simulations } \\
\hline & & $\hat{\pi}$ & $\begin{array}{l}\bar{b} / \tilde{\pi}^{\mathrm{a}} \\
(\%)\end{array}$ & $\begin{array}{l}\hat{\sigma} / \tilde{\pi}^{\mathrm{b}} \\
(\%)\end{array}$ & $E[\pi]$ & $\begin{array}{c}E[b] / \bar{\pi} \\
(\%)\end{array}$ & $\begin{array}{c}E[\sigma] / \tilde{\pi} \\
(\%)\end{array}$ \\
\hline 200 & $1 \cdot 50$ & $1 \cdot 47$ & $-0 \cdot 28$ & $1 \cdot 72$ & $1 \cdot 50$ & -0.02 & $1 \cdot 81$ \\
\hline 30 & $10 \cdot 0$ & $9 \cdot 37$ & $+1 \cdot 41$ & $8 \cdot 33$ & $10 \cdot 1$ & $+1 \cdot 22$ & $10 \cdot 5$ \\
\hline 20 & $15 \cdot 0$ & $15 \cdot 3$ & $+2 \cdot 87$ & $15 \cdot 4$ & $15 \cdot 4$ & $+2 \cdot 67$ & $16 \cdot 7$ \\
\hline 10 & $30 \cdot 0$ & $29 \cdot 3$ & $+5 \cdot 39$ & $28 \cdot 0$ & $34 \cdot 6$ & $+15 \cdot 3$ & 137 \\
\hline 5 & $60 \cdot 0$ & 408 & $+3 \times 10^{2}$ & $3 \times 10^{3}$ & 122 & $+1 \times 10^{2}$ & $1 \times 10^{3}$ \\
\hline
\end{tabular}

${ }^{a}$ Estimated by equation (23).

b Estimated by equation (24). 
Table 8. Comparison of relative (MSE) ${ }^{1 / 2}$ for eigenvalue of diluted component for various decompositions. The reported values (in $\%$ ) are the Monte Carlo estimates. The numbers printed bold indicate the limit of detection

\begin{tabular}{rccc}
\hline \multicolumn{1}{r}{$H$} & $\mathbf{N}$ & $\mathbf{N}+\mathbf{M}$ & $(\mathbf{N} \mid \mathbf{M})$ and $\left(\frac{\mathbf{N}}{\mathbf{M}}\right)$ \\
\hline 200 & $2 \cdot 27$ & $1 \cdot 10$ & $1 \cdot 81$ \\
30 & $10 \cdot 0$ & $12 \cdot 4$ & $10 \cdot 6$ \\
20 & $14 \cdot 8$ & $19 \cdot 7$ & $16 \cdot 9$ \\
10 & $29 \cdot 6$ & $\mathbf{4 1 \cdot 8}$ & $\mathbf{1 3 8}$ \\
5 & $58 \cdot 3$ & $87 \cdot 4$ & $1 \times 10^{3}$ \\
\hline
\end{tabular}

first row is comparable with the standard error found for Lorber's method. The bias is, however, much smaller, which is inferred from the measured value in the last column. For the other dilutions the variance is about the same as for the other models and the bias takes values that are comparable with those found for Lorber's method. Here the detection limit is accompanied by a much more drastic increase in the variance and bias. This extreme behavior is also probably caused by the large size of the eigenvalues.

In order to compare the quantitative results for the three models, we have calculated the MSE based on the results for the extensive simulations. (The simulations must be expected to approach the true values.) The values for the MSE are given in Table 8. For the highest dilution, i.e. for major components, the generalization of Sánchez and Kowalski comes out best. For the other dilutions Lorber's method gives the lowest MSE. The results for the generalization of Sánchez and Kowalski can, however, easily be improved by applying the proposed bias correction. The bias estimates seem to be reliable enough (error less then 10\%) for all cases above the detection limit. The second dilution has in fact already been treated as a simple example in the section on bias correction with slightly altered numbers. Using the bias correction for the first three dilutions will finally give the best results for the generalization of Sánchez and Kowalski.

Finally we give in Table 9 the normalized inner products of the reconstructed profiles and the corresponding standards near the detection limit of the diluted component. It is seen that the recognition is excellent even for the diluted component, which is somewhat misleading, since the eigenvalue carries such a large error. It is seen that the spectra are slightly more precise than the elution profiles, as expected. The reconstructed profiles seem to be best for the generalization of Wilson et al., but the differences are very small.

Table 9. Summary of qualitative solution near limit of detection $(H=10)$ : normalized inner products of reconstructed UV spectra and elution profiles and their corresponding standards

\begin{tabular}{|c|c|c|c|c|c|c|c|}
\hline \multirow[b]{2}{*}{ Component } & \multirow[b]{2}{*}{ Standard } & \multicolumn{2}{|c|}{ Decomposition of $\mathbf{N}$} & \multicolumn{2}{|c|}{$\begin{array}{l}\text { Decomposition of } \\
\qquad \mathbf{N}+\mathbf{M}\end{array}$} & \multicolumn{2}{|c|}{$\begin{array}{l}\text { Decomposition of } \\
(\mathbf{N} \mid \mathbf{M}) \text { and }\left(\frac{N}{M}\right)\end{array}$} \\
\hline & & $\begin{array}{c}\text { UV } \\
\text { spectrum }\end{array}$ & $\begin{array}{l}\text { Elution } \\
\text { profile }\end{array}$ & $\begin{array}{l}\text { UV } \\
\text { spectrum }\end{array}$ & $\begin{array}{l}\text { Elution } \\
\text { profile }\end{array}$ & $\begin{array}{l}\text { UV } \\
\text { spectrum }\end{array}$ & $\begin{array}{l}\text { Elution } \\
\text { profile }\end{array}$ \\
\hline 1 & M & $1 \cdot 00000$ & 0.99994 & $1 \cdot 00000$ & 0.99965 & $1 \cdot 00000$ & 0.99995 \\
\hline 2 & $\alpha-\mathrm{C}$ & 0.99999 & 0.99998 & 0.99998 & 0.99992 & $1 \cdot 00000$ & 0.99999 \\
\hline 3 & $\mathrm{C}$ & $1 \cdot 00000$ & 0.99994 & $1 \cdot 00000$ & 0.99998 & $1 \cdot 00000$ & 0.99999 \\
\hline
\end{tabular}




\section{Effect of dimensionality on the error estimates}

The choice of the true dimensionality is in general not a trivial problem. Therefore it is interesting to investigate how the error estimates behave when we underspan or overspan the factor space. Another reason for performing these simulations is the following. With collinear data a good alternative to multiple linear regression (MLR) is principle component regression (PCR), i.e. replace the original regressors by a selection of PCs. Underfactoring automatically introduces a bias, but since the variance is reduced at the same time very often the MSE is decreased. Thus there is a good reason for deliberately underfactoring the model. We will confine the discussion to Lorber's method.

We have simulated a system where the unknown sample contains three components and the calibration sample only one. The peak heights are given in Table 10 . With only one component in the calibration sample the identity of the eigenvalue is easily established even if the profiles are distorted. In practice, distorted profiles may resemble the profiles of other components if the library is large enough, leading to a false identification. The results are given in Table 11. It is seen, that the estimated eigenvalues are severely biased if the model is underfactored. (An instructive example is also given by Malinowski and Howery. ${ }^{19}$ ) Predicted variance and bias (resulting from measurement noise) are extremely small. The predicted variance is in good agreement with the Monte Carlo result. (It should be kept in mind that the symbols in equation (10) no longer correspond to true profiles for the transformed noise factors.) We have no equivalent values for the bias, since the bias is calculated with respect to the true value. The resulting bias is therefore automatically the bias resulting from underfactoring. Increasing the dimensionality from three to five gives only small changes in the eigenvalues, variance and bias respectively. Since the maximum error in the predicted MSE $(3 \cdot 7 \%)$ is found to be $0 \cdot 5 \%$ for

Table 10. Peak heights in mAU for standard and unknown sample

\begin{tabular}{lrrr}
\hline Sample & M & $\alpha-\mathrm{C}$ & \multicolumn{1}{c}{ C } \\
\hline Standard & 0 & 300 & 0 \\
Unknown & 100 & 200 & 300 \\
\hline
\end{tabular}

Table 11. Summary of quantitative solution for analyte of interest for varying dimension of model: decomposition of $\mathbf{M}$. The numbers printed bold indicate the correct dimension

\begin{tabular}{|c|c|c|c|c|c|c|}
\hline \multirow[b]{2}{*}{ Dimension } & \multicolumn{3}{|c|}{ One simulation } & \multicolumn{3}{|c|}{$10^{4}$ simulations } \\
\hline & $\tilde{\pi}$ & $\begin{array}{l}\hat{b} \mid \tilde{\pi}^{\mathbf{a}} \\
(\%)\end{array}$ & $\begin{array}{l}\hat{\sigma} / \tilde{\pi}^{\mathrm{b}} \\
(\%)\end{array}$ & $E[\pi]$ & $\begin{array}{c}E[b] / \tilde{\pi} \\
(\%)\end{array}$ & $\begin{array}{c}E[\sigma] / \tilde{\pi} \\
(\%)\end{array}$ \\
\hline 1 & 0.51 & $-0 \cdot 0$ & $1.5 \times 10^{-3}$ & 0.51 & - & $2 \cdot 3 \times 10^{-3}$ \\
\hline 2 & 0.92 & $-0 \cdot 1$ & $0 \cdot 2$ & 0.92 & - & $0 \cdot 3$ \\
\hline 3 & $1 \cdot 42$ & $-2 \cdot 8$ & $1 \cdot 5$ & $1 \cdot 45$ & $-3 \cdot 4$ & $1 \cdot 6$ \\
\hline 4 & $1 \cdot 47$ & $-3 \cdot 8$ & $1 \cdot 8$ & $1 \cdot 45$ & $-3 \cdot 3$ & $1 \cdot 6$ \\
\hline 5 & $1 \cdot 47$ & $-3 \cdot 5$ & $1 \cdot 7$ & $1 \cdot 45$ & $-3 \cdot 3$ & $1 \cdot 6$ \\
\hline
\end{tabular}

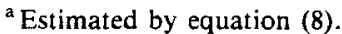

${ }^{b}$ Estimated by equation (24).
} 


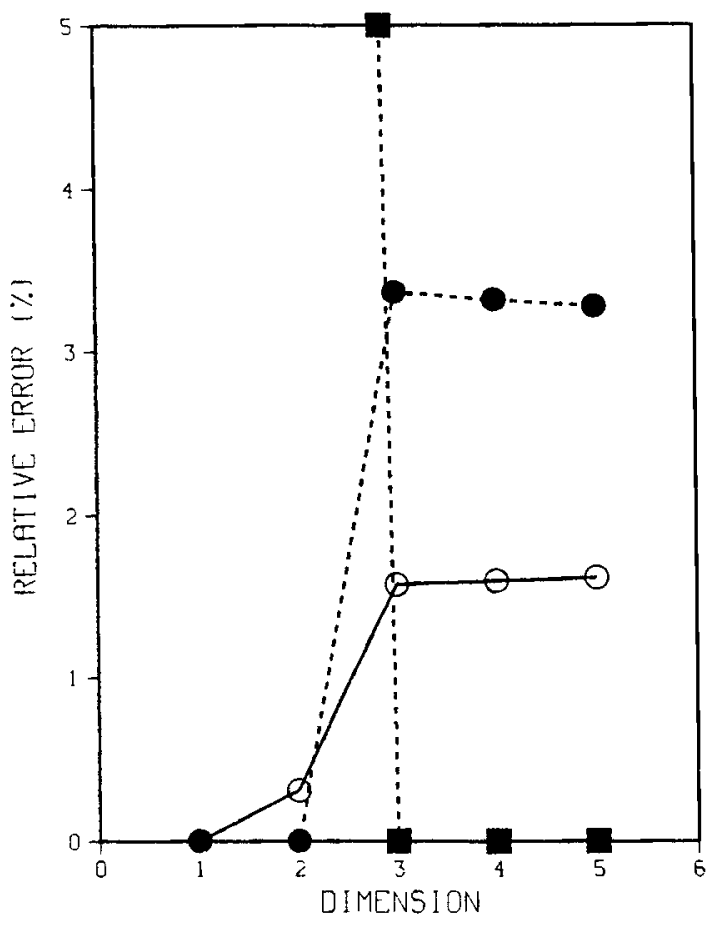

Figure 3. Contribution to mean squared error in the eigenvalues: standard error $(\mathrm{O}-\mathrm{O})$, bias from under factoring ( $\mathbf{a}-\ldots$ ) and bias from measurement noise

the three-dimensional model, it seems that the predictions work equally well for the overdimensioned model. The different contributions to the MSE are depicted in Figure 3. For rank annihilation we always have variance and bias as a result of measurement noise. We usually find that the underfactoring bias is much larger than the reduction in the other contributions. This means that a PCR version of rank annihilation will only give an improved MSE under very extreme circumstances, since the examples worked out here were constructed to represent difficult cases.

\section{CONCLUSIONS}

Expressions have been derived for predicting the bias and variance in the eigenvalues of rank annihilation. In order to derive these expressions, extensive use has been made of analogies between a reformulation of the characteristic eigenvalue problem and the prediction equations of univariate and multivariate calibration. An important difference between the derivation of the bias and variance expressions is the fact that in order to derive the bias expressions, additional assumptions are needed. This is not the case for the variance expressions, where simple first-order error propagation is applied. The additional approximation is probably the reason why in general the bias estimate is found to be less accurate than the variance estimate. A bias correction technique has been proposed that effectively eliminates the bias if the error in the bias is not too large. The simulations discussed in this paper show that probably even an error in the predicted bias as large as $10 \%$ can be tolerated. Because the bias correction 
technique introduces uncertainties, it should, however, be used with care. The necessary steps for establishing confidence limits for the actual concentration ratios have been indicated. It has been demonstrated theoretically and by performing simulations that large differences may occur in variance as well as bias for Lorber's method, the generalization of Sánchez and Kowalski and the generalization of Wilson et al. The generalization of Sánchez and Kowalski should always give the smallest variance. However, the bias increases quickly for this method when the eigenvalue becomes small. The best results are obtained if the concentrations are approximately equal in the unknown and calibration sample (large variance reduction and almost no bias). Lorber's method and the generalization of Wilson et al. behave similarly with respect to variance. Furthermore, both methods display only a moderate increase in bias when approaching the detection limit. The derived expressions also perform well if the dimension of the factor space is not correct. It is found that the bias resulting from under factoring is usually much larger than the decrease in bias and variance resulting from measurement noise. This means that a PCR version of rank annihilation will only work in very exceptional cases. A treatment of other subjects, e.g. wavelength selection, should be equally straightforward using the reformulation of the eigenvalue problem.

\section{APPENDIX I: THE INFLUENCE OF RANDOM NOISE IN UNIVARIATE AND MULTIVARIATE CALIBRATION}

Some important expressions for bias and variance are summarized for univariate and multivariate calibration. These expressions are useful for the derivation and interpretation of similar results for bilinear calibration. The usual simplifying assumptions about noise are made. These simplifications are not necessary and additional results can be found in the literature. ${ }^{6,8,10}$

\section{Univariate calibration (scalar data)}

With errors in both the instrumental response, denoted by $r$, and the sensitivity of the instrument, denoted by $s$, the prediction equation is

$$
\tilde{r}+\delta r=(\tilde{s}+\delta s)(\tilde{c}+\delta c)
$$

where $c$ is the unknown concentration estimated as

$$
\hat{c}=s^{-1} r
$$

If the errors in $r$ and $s$ are sufficiently small, the bias ${ }^{8}$ and variance are given by

$$
\begin{gathered}
\operatorname{bias}(\hat{c})=\tilde{s}^{-2} \tilde{c} \sigma_{s}^{2} \\
\operatorname{var}(\hat{c})=\tilde{s}^{-2}\left(\sigma_{r}^{2}+\tilde{c}^{2} \sigma_{s}^{2}\right)
\end{gathered}
$$

and combined in the MSE as

$$
\operatorname{MSE}(\hat{c})=\tilde{s}^{-2}\left[\sigma_{r}^{2}+\tilde{c}^{2} \sigma_{s}^{2}\left(1+\tilde{s}^{-2} \sigma_{s}^{2}\right)\right]
$$

In equation (43) the relative importance of variance and bias as first- and second-order properties of an estimator become apparent. 


\section{Multivariate calibration (vector data)}

With errors in both the $J \times 1$ response vector, denoted by $\mathbf{r}$, and the $J \times K$ matrix of sensitivities, denoted by $\mathbf{S}$, the prediction equation is

$$
\tilde{\mathbf{r}}+\delta \mathbf{r}=(\tilde{\mathbf{S}}+\delta \mathbf{S})(\tilde{\mathbf{c}}+\delta \mathbf{c})
$$

where $\mathrm{c}$ is the $K \times 1$ vector of unknown concentrations estimated as

$$
\hat{\mathbf{c}}=\mathbf{S}^{+} \mathbf{r}
$$

With errors in $\mathbf{S}$ the estimated concentration is biased, because the pseudoinverse of a stochastic matrix is involved in the calculation. The expectation value of the pseudoinverse matrix $\mathrm{S}^{+}$is given (for the full-rank case) by ${ }^{6}$

$$
\begin{aligned}
E\left[\mathbf{S}^{+}\right] & =E\left[(\tilde{\mathbf{S}}+\delta \mathbf{S})^{+}\right] \\
& =\left[\left[(\tilde{\mathbf{S}}+\delta \mathbf{S})^{\mathrm{T}}(\tilde{\mathbf{S}}+\delta \mathbf{S})\right]^{-1}(\tilde{\mathbf{S}}+\delta \mathbf{S})^{\mathrm{T}}\right] \\
& =\left(\tilde{\mathbf{S}} \tilde{\mathbf{S}}+E\left[\delta \mathbf{S}^{\mathrm{T}} \delta \mathbf{S}\right]\right)^{-1} E\left[\tilde{\mathbf{S}}^{\mathrm{T}}+\delta \mathbf{S}^{\mathrm{T}}\right] \\
& =\left[\mathbf{I}+\left(\tilde{\mathbf{S}}{ }^{\mathrm{T}} \tilde{\mathbf{S}}\right)^{-1} E\left[\delta \mathbf{S}^{\mathrm{T}} \delta \mathbf{S}\right]\right]^{-1} \tilde{\mathbf{S}}^{+}
\end{aligned}
$$

which can be rewritten as

$$
\begin{aligned}
E\left[\mathbf{S}^{+}\right] & =\left(\mathbf{I}+J \tilde{\mathbf{\Phi}} \sigma_{\mathrm{S}}^{2}\right)^{-1} \tilde{\mathbf{S}}^{+} \\
& =\left[\mathbf{I}-(J-K-1) \tilde{\mathbf{\Phi}} \sigma_{\mathrm{S}}^{2}\right] \tilde{\mathbf{S}}^{+}
\end{aligned}
$$

where the substitutions $\left(\mathbf{S}^{\mathrm{T}} \mathbf{S}\right)^{-1}=\Phi$ and $E\left[\delta \mathbf{S}^{\mathrm{T}} \delta \mathbf{S}\right]=J \sigma_{\text {S }}^{2} \mathbf{I}$ have been made. The final expression is corrected for degrees of freedom. ${ }^{6}$ The variance in the $n$th concentration is given by $^{6}$

$$
\operatorname{var}\left(\hat{c}_{n}\right)=\tilde{\Phi}_{n n}\left(\sigma_{\mathrm{r}}^{2}+\|\tilde{c}\|^{2} \sigma_{\mathrm{S}}^{2}\right)
$$

For small errors the off-diagonal elements of the matrix in square brackets in equation (47) contribute very little. Consequently the bias in the determined concentrations can be estimated very well by taking only the diagonal elements into account. This additional approximation leads to the following expression for the MSE:

$$
\operatorname{MSE}\left(\hat{c}_{n}\right)=\tilde{\boldsymbol{\Phi}}_{n n}\left\{\sigma_{\mathrm{r}}^{2}+\|\tilde{c}\|^{2} \sigma_{\mathrm{S}}^{2}+(J-K-1)^{2} \Phi_{n n} \tilde{c}_{n}^{2} \sigma_{\mathrm{S}}^{4}\right\}
$$

It is seen that both bias and variance are primarily influenced by the same quantity, i.e. the corresponding diagonal element of the matrix $\boldsymbol{\Phi}$. This number is usually referred to as the 'variance factor', 11 but it follows that it could also be called the 'bias factor' or more generally the 'MSE factor'.

As mentioned by other researchers, some useful transcriptions are possible for this quantity. Summarizing their results gives

$$
\begin{aligned}
\Phi_{n n} & =\left(\mathbf{S}^{\mathrm{T}} \mathbf{S}\right)_{n n}^{-1} \\
& =\left\|\mathbf{S}_{n \text {-row }}^{+}\right\|^{2} \\
& =\sum_{p=1}^{K}\left(\frac{V_{n p}}{\theta_{\mathrm{S}, p}}\right)^{2} \\
& =(\mathrm{SEN})_{n}^{-2}
\end{aligned}
$$

The identity of (50a) and (50b) has been proved by Bauer et al., ${ }^{10}$ thereby establishing the relation between Lorber's figures of merit ${ }^{12}$ and well-known statistical results. Equation (50c) 
is based on the SVD of $\mathbf{S}: \mathbf{S}=\mathbf{U}_{\mathrm{S}} \boldsymbol{\theta}_{\mathrm{S}} \mathbf{V}_{\mathrm{S}}^{\mathrm{T}}$. It has been used to define so-called variance inflation factors. These factors are useful as a diagnostic for identifying multicollinearities in $\mathbf{S}$. It is a difficult problem as to whether column scaling of $\mathbf{S}$ should be applied. ${ }^{20,21}$ Equation (50d) represents Lorber's definition of multivariate sensitivity. Inserting this symbol in the relevant expressions supplies the correspondence between univariate and multivariate calibration.

\section{APPENDIX II: DERIVATION OF VARIANCE IN THE RECONSTRUCTED PROFILES}

First the reconstructed column profiles are written as

$$
\tilde{\mathbf{H}}+\delta \mathbf{H}=(\tilde{\mathbf{A}}+\delta \mathbf{A})(\tilde{\mathbf{T}}+\delta \mathbf{T})
$$

If the errors in the transformation matrix, $\delta \mathbf{T}$, are small, the errors in the reconstructed column profiles can be approximated by ${ }^{22}$

$$
\delta \mathbf{H}=\delta \mathbf{A} \tilde{\mathbf{T}}
$$

In a previous paper ${ }^{7}$ we showed that the errors in the scores can be approximated by

$$
\delta \mathbf{A}=\tilde{\mathbf{U}} \delta \boldsymbol{\theta}
$$

The resulting standard errors were in good agreement with the Monte Carlo values, but using these expressions in order to derive standard errors in the eigenvalues yielded inconsistent results. Thus using this additional approximation may result in a prediction of the bias that is less accurate than the prediction of the variance. Combining equations (52) and (53) and using $\delta \boldsymbol{\Theta}=\tilde{\mathbf{U}}^{\mathrm{T}} \delta \mathbf{M} \tilde{\mathbf{V}}^{7}$ gives

$$
\begin{aligned}
E\left[\delta \mathbf{H}^{\mathrm{T}} \delta \mathbf{H}\right] & =E\left[\tilde{\mathbf{T}}^{\mathrm{T}} \delta \mathbf{A}^{\mathrm{T}} \delta \mathbf{A} \tilde{\mathbf{T}}\right] \\
& =\tilde{\mathbf{T}}^{\mathrm{T}} E\left[\delta \boldsymbol{\Theta}^{\mathrm{T}} \tilde{\mathbf{U}}^{\mathrm{T}} \tilde{\mathbf{U}} \delta \boldsymbol{\theta}\right] \tilde{\mathbf{T}} \\
& =\tilde{\mathbf{T}}^{\mathrm{T}} \tilde{\mathbf{V}}^{\mathrm{T}} E\left[\delta \mathbf{M}^{\mathrm{T}} \tilde{\mathbf{U}} \tilde{\mathbf{U}}^{\mathrm{T}} \delta \mathbf{M}\right] \tilde{\mathbf{V}} \tilde{\mathbf{T}} \\
& =S \sigma_{\mathbf{M}}^{2}\left(\tilde{\mathbf{T}}^{\mathrm{T}} \tilde{\mathbf{T}}\right)
\end{aligned}
$$

The reconstructed column profiles are correlated and consequently the covariance matrix is not diagonal. This is a difference compared with the multivariate situation. Furthermore,

$$
\left(\tilde{\mathbf{H}}^{\mathrm{T}} \tilde{\mathbf{H}}\right)^{-1}=\tilde{\mathbf{T}}^{-1} \tilde{\boldsymbol{\Lambda}}^{-1} \tilde{\mathbf{T}}^{-\mathrm{T}}
$$

where $\Lambda=\Theta^{2}$. Combining equations (54) and (55) leads to

$$
\left(\tilde{\mathbf{H}}^{\mathrm{T}} \tilde{\mathbf{H}}\right)^{-1} E\left[\delta \mathbf{H}^{\mathrm{T}} \delta \mathbf{H}\right]=S \sigma_{M}^{2}\left(\tilde{\mathbf{T}}^{-1} \tilde{\mathbf{\Lambda}}^{-1} \tilde{\mathbf{T}}\right)
$$

and using equivalent expressions for $\mathbf{Y}$ shows that

$$
E\left[\delta \mathbf{Y}^{\mathrm{T}} \delta \mathbf{Y}\right]\left(\tilde{\mathbf{Y}}^{\mathrm{T}} \tilde{\mathbf{Y}}\right)^{-1}=W \sigma_{M}^{2}\left(\tilde{\mathbf{T}}^{-1} \tilde{\mathbf{\Lambda}}^{-1} \tilde{\mathbf{T}}\right)
$$

It follows that the contributions from the separate modes to the bias in the eigenvalues are intimately related. They are in fact identical for square matrices. It is important to note that this result is derived without any reference to rank annihilation. Therefore it should be valid for any method that gives precise estimates for the transformation matrix $\mathbf{T}$. An immediate consequence of equations (56) and (57) is that the overlap and precision of the reconstructed profiles are complementary. This is important if these profiles are to be used for e.g. identification purposes, as is the case with rank annihilation. For the simulations discussed in 
this paper the spectral mode is much more unselective then the chromatographic mode. If the measurement error were equally dispersed over the two modes, the reconstructed spectra would probably become useless for identification.

\section{APPENDIX III: DERIVATION OF VARIANCE IN THE EIGENVALUES, EXPRESSED IN THE PHYSICAL DECOMPOSITION OF M}

We will only discuss Lorber's method and drop all subscripts to simplify the notation. First equation (7) is rewritten as

$$
(\tilde{\mathbf{H}}+\delta \mathbf{H})^{+}(\tilde{\mathbf{N}}+\delta \mathbf{N})(\tilde{\mathbf{Y}}+\delta \mathbf{Y})^{\ddagger}=\boldsymbol{\Pi}+\delta \boldsymbol{\Pi}
$$

Note that the spread around $\boldsymbol{\Pi}$ instead of $\overline{\boldsymbol{\Pi}}$ is estimated. The error in the pseudoinverse of a matrix $\mathbf{A}+\delta \mathbf{A}$ can be approximated by $(\mathbf{A}+\delta \mathbf{A})^{+}=\mathbf{A}^{+}+\delta\left(\mathbf{A}^{+}\right){ }^{24}$ This results in

$$
\delta \Pi=\tilde{\mathbf{H}}^{+} \delta \mathbf{N} \tilde{\mathbf{Y}}{ }^{\ddagger}+\delta\left(\mathbf{H}^{+}\right) \tilde{\mathbf{N}} \tilde{\mathbf{Y}}^{\ddagger}+\tilde{\mathbf{H}}^{+} \tilde{\mathbf{N}} \delta\left(\mathbf{Y}^{\ddagger}\right)
$$

Using $\delta\left(\mathbf{A}^{+}\right)=-\mathbf{A}^{+} \delta \mathbf{A} \mathbf{A}^{+}$(see Reference 10 ) and commutivity for diagonal matrices yields

$$
\delta \boldsymbol{\Pi}=\tilde{\mathbf{H}}^{+} \delta \mathbf{N} \tilde{\mathbf{Y}}^{\ddagger}-\mathbf{\Pi} \tilde{\mathbf{H}}^{+} \delta \mathbf{M} \tilde{\mathbf{Y}}^{\ddagger}
$$

leading to the following statistical error, if the measurement noise is uncorrelated:

$$
\operatorname{var}\left(\hat{\pi}_{n}\right)=\sum_{i=1}^{r} \sum_{k=1}^{\mathcal{c}}\left(\tilde{H}_{n i}^{+}\right)^{2}\left(\tilde{Y}_{k n}^{\ddagger}\right)^{2}\left(\sigma_{N_{i k}}^{2}+\pi_{n}^{2} \sigma_{M_{i k}}^{2}\right)
$$

This result simplifies considerably if the measurement noise is homoscedastic:

$$
\operatorname{var}\left(\hat{\pi}_{n}\right)=\left\|\tilde{\mathbf{H}}_{n \text {-row }}^{+}\right\|^{2}\left\|\tilde{\mathbf{Y}}_{n \text {-col }}^{+}\right\|^{2}\left(\sigma_{\mathrm{N}}^{2}+\pi_{n}^{2} \sigma_{\mathrm{M}}^{2}\right)
$$

leading through equation (10) to equation (24). The corresponding equation for the generalization of Sánchez and Kowalski is derived by working out (62) after making the necessary substitutions.

\section{REFERENCES}

1. C.-N. Ho, G. D. Christian and E. Davidson, Anal. Chem. 50, 1108 (1978).

2. N. M. Faber, L. M. C. Buydens and G. Kateman, J. Chemometrics, 8, 147 (1994).

3. A. Lorber, Anal. Chim. Acta, 164, 293 (1983).

4. E. Sánchez and B. R. Kowalski, Anal. Chem. 58, 496 (1986).

5. B. E. Wilson, E. Sánchez and B. R. Kowalski, J. Chemometrics, 3, 493 (1989).

6. S. D. Hodges and P. G. Moore, Appl. Stat. 21, 185 (1972).

7. N. M. Faber, L. M. C. Buydens and G. Kateman, J. Chemometrics, 7, 495 (1993).

8. M. G. Moran and B. R. Kowalski, Anal. Chem. 56, 562 (1984).

9. H. L. Gray and W. R. Schucany, The Generalized Jackknife Statistic, Marcel Dekker, New York (1972).

10. G. Bauer, W. Wegscheider and H. M. Ortner, Spectrochim. Acta B, 46, 1185 (1991).

11. J. Mandel, J. Res. NBS, 90, 465 (1985).

12. A. Lorber, Anal. Chem. 58, 1167 (1986).

13. C.-N. Ho, G. D. Christian and E. R. Davidson, Anal Chem. 52, 1071 (1980).

14. C. J. Appelof and E. R. Davidson, Anal. Chim. Acta, 146, 9 (1983).

15. W. A. Fuller, Measurement Error Models, Wiley, New York (1987).

16. P. J. Bickel and K. A. Doksum, Mathematical Statistics, Holden-Day, San Fransisco, CA (1977).

17. G. Bauer, W. Wegscheider and H. M. Ortner, J. Anal. Chem. 340, 135 (1991).

18. B. G. M. Vandeginste, F. Leyten, M. Gerritsen, J. W. Noor and G. Kateman, J. Chemometrics, I, 57 (1987) 
19. E. R. Malinowski and D. G. Howery, Factor Analysis in Chemistry, Wiley, New York (1991).

20. P. K. Hopke, Receptor Modeling in Environmental Chemistry, Wiley, New York, (1985).

21. J. H. Kalivas, J. Chemometrics, 3, 489 (1989).

22. E. R. Malinowski, Anal. Chim. Acta, 122, 327 (1980).

23. B. A. Roscoe and P. K. Hopke, Anal. Chim. Acta, 135, 379 (1982).

24. T. V. Karstang, J. Toft and O. M. Kvalheim, J Chemometrics, 6, 177 (1992). 